Journal of

Neurophysiology and Neurological Disorders

\title{
What Can Attention Abilities Teach Us about Reading Comprehension in NF1?
}

Maëlle Biotteau ${ }^{1 *}$, Elodie Tournay ${ }^{2}$, Eloise Baudou ${ }^{1,3}$, Sandrine Lelong ${ }^{4}$, Stéphanie Iannuzzi ${ }^{3}$, Nathalie FaureMarie $^{3}$, Pierre Castelnau ${ }^{5,6,7}$, Elisabeth Schweitzer ${ }^{7}$, Diana Rodriguez ${ }^{8,9,10}$, Isabelle Kemlin ${ }^{8}$, Nathalie Dorison ${ }^{8}$, François Rivier ${ }^{11,12}$, Maryline Carneiro ${ }^{11}$, Elodie Preclaire ${ }^{12}$, Sebastien Barbarot ${ }^{13}$, Valérie Lauwers-Cancès ${ }^{2}$, Yves Chaix ${ }^{1,3}$

${ }^{1}$ ToNIC, Toulouse NeuroImaging Center, University of Toulouse, Inserm, UPS, France

${ }^{2}$ Epidemiology Department, Toulouse University Hospital, Toulouse, France

${ }^{3}$ Children's Hospital, Toulouse-Purpan University Hospital, Toulouse, France

${ }^{4}$ Pediatric Clinical Research Unit, Toulouse Clinical Investigation Center, Children's Hospital, Purpan University Hospital, Toulouse, France

${ }^{5}$ Brain \& Imaging Joint Research Unit (UMR 930), Bretonneau Hospital, Tours Regional University Hospital, Tours, France ${ }^{6}$ University of Tours François Rabelais, Tours, France

${ }^{7}$ Neuropediatrics \& Disabilities Unit, Gatien de Clocheville Children's Hospital, Tours University Hospital, Tours, France

${ }^{8}$ Pediatric Neurology Department \& Neurofibromatosis Referral Center, Armand Trousseau Hospital, East Paris University Hospital, Paris, France

${ }^{9}$ University of Paris VI Pierre \& Marie Curie, Sorbonne Universities, Paris, France

${ }^{10}$ Neuroprotection of the Developing Brain Joint Research Unit (U1141), INSERM, Paris, France

${ }^{11}$ Department of Pediatric Neurology, CHU Montpellier, PhyMedExp, University of Montpellier, INSERM, CNRS, Montpellier, France

${ }^{12}$ Department of Pediatric Neurology \& Reference Center for language disabilities, CHU Montpellier, France

${ }^{13}$ Dermatology Clinic, Hôtel-Dieu University Hospital, Nantes, France

${ }^{*}$ Corresponding author: Maelle Biotteau, UMR 1214 - Inserm/UPS - ToNIC, Toulouse NeuroImaging Center - CHU PURPAN Pavillon Baudot - Place du Dr Baylac - 31024 TOULOUSE - Cedex 3, France, E-mail: maelle.biotteau@inserm.fr

Received Date: September 06, 2019 Accepted Date: October 16, 2019 Published Date: October 19, 2019

Citation: Maëlle Biotteau (2019) What Can Attention Abilities Teach Us about Reading Comprehension in NF1? J Neurophysiol Neurol Disord 5: 1-17.

\section{Abstract}

Attention span impacts reading quality in many different settings and it is also one of the major cognitive disorders in neurofibromatosis type 1 (NF1). The current study aimed to evaluate the impact of attention on reading comprehension, in NF1 and non-NF1children. A multicenter, cross-sectional study was conducted in two groups of 150 children (8-12yo)

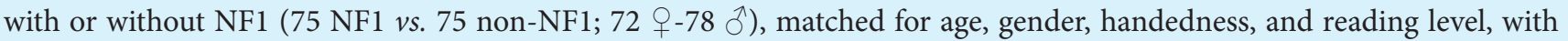
reading level considered as a continuum ranging from good to poor readers. Children with intellectual deficiencies, neurological and psychiatric disorders were excluded from the study. Attention skills were assessed with a parental questionnaire $(\mathrm{CBCL})$ as well as a performance-based evaluation (CPT-II). Reading comprehension was assessed with a standardized reading comprehension test. The attention performance-based scores assessed text and sentence comprehension between the two groups ( $\mathrm{p}=0.0235$ and $\mathrm{p}=0.0164$, respectively), whereas indirect questionnaire attention scores only measured sentence comprehension $(\mathrm{p}=0.0263)$. Both groups exhibited weak correlations between questionnaire and performance-based scores. We show that reading comprehension is greatly influenced by attention in both NF1 and non-NF1 groups, even when predictors of good reading comprehension, such as IQ score and reading accuracy, are included in the analysis. Indirect observer-rated (questionnaires) and direct performance-based evaluations of attention measure distinct factors, associated with different components of reading skills, and are not interchangeable assessments of attention difficulties. Both assessments are complementary and should be used in parallel for a multimodal assessment of attention.

C2019 The Authors. Published by the JScholar under the terms of the Creative Commons Attribution License http://creativecommons.org/licenses/ by/3.0/, which permits unrestricted use, provided the original author and source are credited. 
Keywords: NF1; Dyslexia; Child; ADHD; Reading Processes; Attention Processes; Assessment; Tests

\section{Introduction}

\section{Prevalence \& Physical Characteristics of NF1}

Neurofibromatosis type $1(\mathrm{NF} 1)$ is one of the most common childhood genetic disorders, affecting approximately 1 in 2500 to 3000 individuals in the general population [1]. The diagnosis of NF1 is made based on physical characteristics as stated in the 1988 National Institutes of Health Consensus Development Conference Statement, and its 1997 update [2], which includes cutaneous, ophthalmologic, neurologic and orthopedic features [3].

\section{Cognitive Phenotype and Learning Disabilities in NF1 Cognitive Deficits}

A broad spectrum of cognitive deficits occurs in 30$70 \%$ of cases [4]. Studies focusing on the neuropsychological phenotype of NF1 children detect a left shift in average IQ, ranging from low to normal IQs [5], and specific deficits in several domains: attention processes, executive function, language acquisition, visuospatial and visual perception [6], and phonology $[4,7,8]$, with the severity of these deficits varying greatly from one child to the next.

NF1 cognitive problems also have the greatest impact on the quality of life $[9,10]$. They significantly impact academic performance [11,12] in particular reading [13] but also arithmetic and written expression. But, the specific contribution of attention disorders to academic difficulties has to date not been investigated.

\section{Potential Causes of NF1 Reading Impairment}

Studies of reading skills in NF1 have predominantly investigated discrepancies in IQ-achievements $[4,14,15]$. When compared to age-matched controls, Cutting et al. (2000) found that NF1 children had reading deficits which specifically affected (i) single-word reading and reading comprehension, (ii) letter-word identification and (iii) passage comprehension (as well as other skills associated with reading achievements such as rapid naming and phoneme segmentation). It has been estimated that between 50\% [16] and 67\% [15] of NF1 children suffer from reading deficits. Watts et al. (2008) further specified that $50 \%$ of NFI children satisfy the phonological dyslexia criteria and $13.3 \%$ of the mixed dyslexia criteria.

Reading is a multifaceted skill consisting of two primary components: word recognition and comprehension, with only one of these components, or both components impaired. Different cognitive deficits may contribute to reading difficulties, specifically oral comprehension, working memory, phonological skills [8], visual perception [14], but also attention processes. Considered one of the primary cognitive concerns in NF1 children $[17,18,19]$, attention skills are a major contributor to reading impairment.

\section{The Impact of Attention Skills on Reading in NF1 Attention Skills and Reading in ADHD Children and the General Population}

It is now well-documented that elevated levels of inattention and/or hyperactivity/impulsivity are associated with various difficulties in the academic domain, including reading ability. This association has not only been confirmed in general populations of preschoolers [20], school-aged children [21,22] but also in adults [23]. Children with impaired attention, but who do not satisfy the attention deficit hyperactivity disorder (ADHD) criteria per se, have also been found to have reading problems $[24,25]$. It is widely accepted that there is a reciprocal causal relationship between attention and reading, where difficulties in one area intensify difficulties in the other [26].

Comorbid reading difficulties are also estimated to affect between 15 to $30 \%$ of ADHD children [27]. Indeed, ADHD children obtain lower reading comprehension scores compared to their peers without ADHD, which extends to all individual score parameters, such as the ability to read words, word identification, reading speed, vocabulary, cognitive skills, and background knowledge [28,29].

Furthermore, children with attention difficulties have lower reading comprehension scores, more difficulty reporting the central idea of a passage and their reading comprehension decreases proportionally with the increase in reading time length - potentially due to the attentional demands of reading lengthy passages which require more processing efforts [28].

\section{Attention Skills and Reading in NF1 Children}

In contrast to ADHD, very little is known about any potential associations between inattentive and hyperactive/impulsive behaviors and reading comprehension-related skills in the NF1 population. Even though $40 \%$ to $50 \%$ of NF1 children satisfy the ADHD diagnostic criteria [4,7,30,31,32], making ADHD one of the principal features of the clinical neurofibromatosis phenotype, the impact of attention skills on cognitive functions of NF1 children has received little coverage. We do however know that NF1 children with ADHD seem to experience more difficulties in terms of IQ, social skills, and some executive functions when compared to NF1 children without ADHD [31,32,33]. But visuospatial deficit $[6,33]$, phonological deficit [8], and some execu- 
tive function impairments $[4,32,34]$ appear to be independent of limitations in attentional abilities.

The literature to date is also vague about how ADHD affects cognitive functions associated with academic achievements. Although ADHD may affect the child's ability to learn, only one study has specifically investigated the association between attention abilities and reading in an NF1 population [35]. The authors showed that children with NF1+ADHD performed significantly worse on reading, spelling, mathematical reasoning, sustained attention, and receptive language, but not intelligence, verbal memory, selective, divided and switching attention and visual-spatial skills compared to the NF1 only group. Furthermore, a backward linear regression model shows that ADHD symptom severity and a performance-based measure of attentional control (TEA-Ch creature counting) were the best predictors of academic achievement (represented by the mean of Reading, Mathematics and Spelling skills) in both the NF1+ADHD and NF1 only groups.

\section{The Complexities of Assessing Attention Skills}

The assessment of attention skills poses substantial challenges. Firstly, there is a large diversity of attention domains that can be assessed using multiple approaches. These approaches can nevertheless be broadly subdivided into two major classes: "indirect observer-rated data" (questionnaires, checklists, interviews, think-aloud protocols, global rating scales, self-report instruments, direct observation of behavior, etc.) and "direct performance-based data" (performance-based measures of functional skills, indices of competence, real-world outcomes). "Indirect observer-rated data" provides a generalized picture of attention performance across everyday functions, in line with the diagnostic criteria defined by the DSM. This type of data is completed by individuals who are expected to make a judgment on a child's behavior (i.e. teachers, parents, etc.). They are the most commonly used forms of assessment (particularly rating scales), predominantly because of their efficiency. Indirect observer-rated data, however, tend to be abstract and introduces the subjective perspective of the respondent (often parents or teachers), ranging from different expectations across environments to inter-rater disagreements, bias, and different behavioral tolerances [36,37]. Rating scales, for example, often have a poor teacher and parent inter-rater agreement $[38,39]$. The extrapolation of results based on indirect observer-rated data therefore requires considerable vigilance. "Direct performance-based data" on the other hand, is collected directly from the children. This approach may provide a more direct and valid estimate of attention abilities. This type of data has the additional benefit of circumventing response bias or can be adapted to capture specific biases. However, performance-based measures of attentional capacity require the subject to actively participate in the assessment process (motivation and cooperation), which is not always evident in practice, especially in children. Moreover, they present an artificial evaluation as they don't capture the child's performance in everyday life settings (the validity of these tests in different environments remains unclear).

In addition, attention is a continuum and should be measured as such. Particularly when identifying pathological $v s$ non-pathological degrees of attention impairment (ADHD vs. non-ADHD) or addressing questions regarding diagnosis or frequency of co-occurrence between two pathologies. Assessments of attention therefore need to take into account that attention develops along a continuum. Inattention, hyperactivity and impulsivity have been consistently proven to be associated with specific domains of daily function or learning, even in children without elevated behavioral problems, and therefore require the child's behavior to be assessed dimensionally [40].

\section{Aims and Objectives}

Reading and attentional impairments occur together in 50 to $70 \%$ of NF1 children. The effect of attention on reading skills, particularly on reading comprehension, is well-accepted in the general population, as well as in populations with specific learning disorders, and in the ADHD population. However, in the case of NF1, the link between attention and reading has to date received little coverage. Although the optimal way to evaluate attention skills is to use both direct and indirect (questionnaires) measures of attention, few previous studies have combined these two types of assessments. Indeed, little work has been done using direct measures of inattention and impulsivity (instead of indirect measure). In addition, few studies have attempted to analyze the association between reading and attention NF1 as a normal variation: all previous studies focused on pathological forms (ADHD and Reading Disorder -RD) vs. non-pathological forms, even though such extremes form part of the continuum. However, if attention is viewed as a component of reading at one end of the curve, the interaction may persist throughout the continuum.

The purpose of this study was to evaluate attention problems using a dimensional approach, combining an assessment from an indirect functional parental questionnaire and a direct neuropsychological assessment of attention in a population of children with NF1 compared to non-NF1 children. Children were matched for gender, age, laterality, and reading level. We first correlated functional (indirect observer-rated) and 
neuropsychological (direct performance-based) assessments of attention. The second objective was to clarify the correlation between reading and attentional variables (questionnaires and tests scores). We subsequently determined which scores measuring attentional abilities were most strongly associated with reading comprehension when taking into account IQ, socio-professional category, and highest education level of parents.

\section{Method \\ Participants}

75 children (78 girls, 72 boys) aged between 8 and 12 years of age with a confirmed diagnosis of NF1 according to the $\mathrm{NIH}$ criteria [2] and 75 control subjects with or without reading difficulties, matched for age (plus or minus 1 year), gender, laterality and reading level (plus or minus 0.5 standard deviation (SD) on the French test "L' Alouette" [41] were recruited through six French national NF1 referral centers in Lyon, Montpellier, Nantes, Paris, Toulouse, and Tours. Peer controls were clinically examined by a neuropediatrician to confirm that they did not satisfy the NF1 diagnostic criteria. In the two groups, children with neurological or psychiatric disorders that could potentially affect cognition (epilepsy, brain tumor, symptomatic optic glioma at MRI, hydrocephalus, head injury, autism or intellectual disability with an IQ below 70) or with uncorrectable hearing or visual impairment were excluded. Treatment with methylphenidate was not an exclusion criterion and, in these cases, the neuropsychological assessment was performed under medication. All parents and children gave their informed oral and written consent. Approval to conduct this study was granted by the French Health Ministry's Hospital Program of Clinical Research (PHRC 2008, Toulouse University Hospital, no. 08113 01), Occitanie Regional Council (APRTC no. 09004813), and the local Ethics Committee (CPP Southwest, France) in accordance with the Declaration of Helsinki.

\section{Procedure}

Participants underwent neuropsychological assessments, using a comprehensive and extensive protocol designed to assess the cognitive level, reading skills, phonological process, visuoperceptual abilities and attention (procedure previously described by Chaix et al. [8].

\section{Measures}

(i) The cognitive assessment included all subtests of the Wechsler Intelligence Scale for Children - Fourth Edition [42]; and the French version of the Peabody Picture Vocabulary Test-Revised (EVIP) [43].

(ii) Reading and phonological skills were assessed with the
"LAAlouette" French reading test [41], the ODEDYS-2 test [44] and the ORLEC battery subtest L1 and L3 [45]. The reliability and validity of these three tests are deemed satisfactory for most measurements [41,44,45]. The "L'Alouette" test assesses the level of lexical decoding, two indices of accuracy and speed when reading a text aloud. These indices were standardized for age to obtain z-scores. Reading accuracy and speed disabilities were defined as a score of at least -1.5SD, below the normative mean value for children of similar ages. The ODEDYS-2 test measured word recognition processes on a series of 20 regular words, 20 irregular words and 20 non-words (pseudo-words), to be read aloud. Both accuracy and speed were considered. This test was used to subdivide the reading profile of participants (e.g., phonological, surface, or mixed dyslexia). The ORLEC battery assessed reading comprehension of sentences as well as texts [45]. The first subtest (L1) consisted of a text to be read aloud. Children answered 6 questions after reading it. The second subtest (L3) consisted of 36 sentences with one word missing from each of the sentences. The children are given 5 choices for the missing word and need to choose the correct word for the individual sentence to make sense. Children have complete as many sentences as they can in 5 minutes. These two subtests provide a global measure of reading comprehension. Children were considered as having a reading comprehension disability if their Lobrot score standardized on normative data for children of the same age was in the fourth quartile.

(iii) The visuo-perceptual assessment was performed using the Judgment of Line Orientation test [46], the Thurstone test and the Corsi blocks.

(iv) Attention skills were assessed with the Conners' Continuous Performance Test-Second Edition (CPT-II) [47] and the parent form of the Child Behavior Checklist (CBCL) questionnaire [48]. The Conners' Continuous Performance Test-Second Edition (CPT- II) [47] computerized test is designed to specifically measure sustained attention and impulsivity. It is generally used for differentiating children with and without inattentive, hyperactive, and impulsive behavioral difficulties $[49,50]$. The test is 14 minutes long and during this time, the child must refrain from pressing the space bar whenever the letter " $\mathrm{X}$ " appears but is required to press the space bar for any other letter (target/non-target stimuli). CPT-II is designed to have minimal language and memory demands. Three percentile scores were used in this analysis: the omissions score (number of non responses to target), the commission's score (number of responses to non-target stimuli) and the Hit RT Standard Error score (a measure of response speed consistency). High percentile scores reflect significant attention problems and the disability level for each dimension was defined as a percentile score above or equal to 90 . The parental 
form of the Child Behavior Checklist (CBCL) [48] questionnaire measure attention problems and psychosocial adjustment. The CBCL "attention problems" subscale which focuses on 11 questions among the 113 in the complete CBCL test was used in this analysis. First, a raw-score was computed as the sum of the 11 scores between 0 and 2, then standardized to obtain the z-score which expresses the number of standard deviations (SD) from the mean in the general population of the same gender and age. Raw-scores $\geq 11$ were considered to indicate "attention problem" disabilities.

\section{Statistical Analysis}

Attentional capacities, reading skills, IQ, and sociocultural characteristics were compared between NF1 and nonNF1 children using the paired Student T-test and the Wilcoxon signed-rank test (when the normality assumption of differences NF1 minus control was rejected) for continuous variables, the McNemar test for binary variables, and the Bowker's test of symmetry for categorical variables with more than 2 categories.

Spearman correlation coefficients were computed to assess the correlations between the CBCL "attention problem" z-score and the CPT-II percentile scores separately, in NF1 and in non-NF1 children, and then on all children with disabilities in CBCL. Significance levels ( $p$-values) were used to assess whether the correlation coefficients were significantly different from zero.

To identify which factors were associated with the text and sentence comprehension scores (Lobrot), a multivariate analysis of covariance was applied to all children. Regressors initially included in the models were CBCL "attention problem" Z-scores, omission, commission percentile scores (CPT-II), EVIP normalized score, IQ and reading speed and accuracy z-scores (L'Alouette). The Hit RT Standard Error percentile score was not used in this multivariate analysis due to its high correlation with omissions which were considered more relevant clinically. Classification variables were the population (NF1 vs. non-NF1) as well as the parents' educational and socio-professional levels. Only children with no missing values for all dependent and independent variables were included in the analysis. A backward elimination procedure starting from the full model was manually applied to remove predictors based on the significance level and stopped when all predictors remaining in the model reached significance, at the 0.05 level. At each step, the parameter estimated changes were checked to detect any possible confounding factors. In the final modeling step, all interactions between the population and the remaining variables were added to the model, which was then reduced using a backward elimination technique on interaction terms to keep only significant interactions, at the 0.10 level. The R-Square $\left(\mathrm{R}^{2}\right)$ of final models was computed to measure how much variation in the text and sentence comprehension scores was accounted for by the predictors.

Statistical tests were two-sided without multiplicity adjustment and all analyses were performed with SAS version 9.4 (SAS Institute Inc., Cary, NC, USA).

\section{Results \\ General Characteristics of the Population}

75 NF1 children and 75 matched controls were included in the study; 72 boys and 78 girls aged between 8 and 12 years of age (mean age of 10 in both groups). The socio-cultural characteristics of the parents' and children's intellectual quotient (IQ) are shown in Table 1. A difference was found between the NF1 and non-NF1 population on parents' education level, with $73.3 \%$ of control parents having a high education level (above high school diploma in the US or BAC in France), compared to $43.2 \%$ of NF1 children parents ( $\mathrm{p}=0.0103)$. The total IQ was also different between groups, with a mean decrease of -9.2 IQ points in NF1 children compared to their paired controls $(\mathrm{p}<0.0001)$.

\section{Attentional Capacity}

The measures of attentional ability are presented in Table 1. The CBCL “attention problem" z-scores were significantly higher in the NF1 children than in the non-NF1 children, with $+0.6 \mathrm{SD}$ on average in NF1 children, indicating greater attention problems in this population ( $\mathrm{p}=0.0421)$. Nineteen NF1 children (25.7\%) and 16 controls (21.6\%) were classified as deficient in attention according to the CBCL $(\mathrm{p}=0.7111)$. The groups were not significantly different for the omissions, commissions and Hit RT Standard Error dimensions of the CPT-II.

\section{Reading Skills}

As shown in Table 1, text and sentence comprehension (Lobrot) were not significantly different between NF1 and controls. While pairs were matched on reading speed, the reading accuracy was significantly worse in NF1 compared to controls (-0.7SD on average, $\mathrm{p}=0.0030)$. However, this difference was not replicated when we considered the precision index as part of the 'pathological' vs. 'non-pathological' percentages, since 27 NF1/75 (36\%) had disabilities in reading accuracy, defined by a z-score $\leq-1.5 \mathrm{SD}$ vs. 18 non-NF1/75 (24\%) ( $\mathrm{p}=0.0636)$ according to the French "LA'Alouette" test. The assessment of reading comprehension skills emphasized oral comprehension, but as shown in Table 1, the EVIP normalized scores were not found to significantly differ between NF1 and controls.

\section{Correlations between CBCL and CPT-II Attention Tests} A weak correlation, between CBCL "attention problem" 
Table 1: Population socio-cultural characteristics, cognitive, attention and reading abilities

\begin{tabular}{|c|c|c|c|c|}
\hline & $\begin{array}{l}\text { NF1 } \\
(\mathrm{N}=75)\end{array}$ & $\begin{array}{l}\text { Controls } \\
(\mathrm{N}=75)\end{array}$ & $\begin{array}{l}\text { Difference } \\
\text { NF1-Control } \\
(\mathrm{N}=75)^{*}\end{array}$ & P-value \\
\hline \multicolumn{5}{|l|}{ Socio-cultural characteristics and general cognitive level } \\
\hline Parents' highest socio-professional category - n (\%) & & & & 0.1102 \\
\hline $\begin{array}{l}\text { Artisan, shopkeeper, company head, } \\
\text { executive or intellectual job }\end{array}$ & $34(45.3 \%)$ & $24(32.0 \%)$ & & \\
\hline Others & $41(54.7 \%)$ & $51(68.0 \%)$ & & \\
\hline Parents' education highest level - n (\%) & & & & 0.0103 \\
\hline Below 12 yo & $23(31.1 \%)$ & $10(13.3 \%)$ & & \\
\hline 12 yo & $19(25.7 \%)$ & $10(13.3 \%)$ & & \\
\hline Above 12 yo & $32(43.2 \%)$ & $55(73.3 \%)$ & & \\
\hline Total IQ: mean (SD) & $89.2(12.03)$ & $98.4(15.06)$ & $-9.2(17.16)$ & $<.0001$ \\
\hline \multicolumn{5}{|l|}{ Attentional capacity } \\
\hline \multicolumn{5}{|l|}{ Attention problems (CBCL): } \\
\hline Z-score - mean (SD) & $1.8(1.58)$ & $1.2(1.66)$ & $0.6(2.37)$ & 0.0421 \\
\hline \multicolumn{5}{|l|}{ Omissions (CPT-II): } \\
\hline Percentile score - mean (SD) & $59.5(24.98)$ & $59.4(25.55)$ & $-1.0(31.82)$ & 0.7920 \\
\hline \multicolumn{5}{|l|}{ Commissions (CPT-II): } \\
\hline Percentile score - mean (SD) & $54.6(26.71)$ & $59.4(25.55)$ & $-5.6(32.98)$ & 0.1624 \\
\hline \multicolumn{5}{|l|}{ Hit RT Std. Error (CPT-II): } \\
\hline Percentile score - mean (SD) & $62.3(28.37)$ & $64.7(25.64)$ & $-3.8(35.28)$ & 0.3781 \\
\hline \multicolumn{5}{|l|}{ Reading skills } \\
\hline \multicolumn{5}{|l|}{ Text reading comprehension (Lobrot): } \\
\hline $\begin{array}{l}\text { Score - mean }(\mathrm{SD}) \\
\text { Sentences reading }\end{array}$ & $72.3(15.45)$ & $69.1(17.87)$ & $2.3(16.05)$ & 0.2301 \\
\hline comprehension (Lobrot): & $21.5(7.68)$ & $20.7(8.97)$ & $0.4(6.95)$ & 0.6385 \\
\hline \multicolumn{5}{|l|}{$\begin{array}{l}\text { Score - mean }(\mathrm{SD}) \\
\text { Reading accuracy (Alouette): }\end{array}$} \\
\hline Z-score - mean (SD) & $-1.6(2.18)$ & $-0.8(1.49)$ & $-0.7(2.06)$ & 0.0030 \\
\hline Disabilities ( $\leq-1.5 \mathrm{SD})-\mathrm{n}(\%)$ & $27(36.0 \%)$ & $18(24.0 \%)$ & & 0.0636 \\
\hline \multicolumn{5}{|l|}{ Reading speed (Alouette): } \\
\hline Z-score - mean (SD) & $-0.6(0.94)$ & $-0.6(1.03)$ & $-0.0(0.35)$ & ND \\
\hline Disabilities ( $\leq-1.5 \mathrm{SD})-\mathrm{n}(\%)$ & $14(18.7 \%)$ & $19(25.3 \%)$ & & 0.1250 \\
\hline EVIP: & & & & 0.1083 \\
\hline Normalized score - mean (SD) & $113.0(14.66)$ & $116.2(14.08)$ & $-3.2(17.19)$ & \\
\hline
\end{tabular}

SD: Standard Deviation; n: number; ND: test not done as reading speed $\mathrm{z}$-score was a matching criterion

${ }^{*} \mathrm{CBCL}$ was assessed for 73 pairs, CPT-II for 69 pairs and Lobrot test for 73 pairs 
z-scores and CPT-II percentiles scores, was detected in both the NF1 and control groups, suggesting that these two tests measure independent factors (Table 2). The highest correlations with the CBCL subscale were observed for omissions $(\rho=0.20, p=0.0865)$ on non-NF1 controls. Because of some missing data in CBCL and CPT-II assessments, correlations were only computed for 71 NF1 children and 71 controls.

Table 2: Spearman correlations between « attention problem » z-scores of CBCL and CPT-II percentiles scores

\begin{tabular}{|l|l|l|}
\hline \multicolumn{2}{|l|}{ « Attention problem » z-scores $(\mathrm{CBCL})$} \\
\hline CPT-II & $\mathrm{NF} 1(\mathrm{~N}=71)$ & Controls $(\mathrm{N}=71)$ \\
\hline Omissions percentile $-\rho(\mathrm{p}$-value $)$ & $0.09(0.4319)$ & $0.20(0.0865)$ \\
\hline Commissions percentile $-\rho(\mathrm{p}$-value $)$ & $-0.10(0.4020)$ & $0.01(0.9133)$ \\
\hline Hit RT Std. Error percentile $-\rho(\mathrm{p}$-value $)$ & $0.13(0.2859)$ & $0.12(0.3069)$ \\
\hline Perseverations percentile $-\rho(\mathrm{p}$-value $)$ & $0.12(0.3029)$ & $0.21(0.0777)$ \\
\hline
\end{tabular}

$\rho:$ correlation coefficient

Table 3: Final models to substantiate reading comprehension scores after selection of variables by backward procedure on all children $(\mathrm{N}=139)$

\begin{tabular}{|c|c|c|c|c|}
\hline \multirow[b]{2}{*}{ Parameters } & \multicolumn{2}{|c|}{$\begin{array}{l}\text { Text comprehension (Lobrot) } \\
\mathrm{R}^{2}=0.46\end{array}$} & \multicolumn{2}{|c|}{$\begin{array}{l}\text { Sentence comprehension (Lobrot) } \\
\mathbf{R}^{2}=0.57\end{array}$} \\
\hline & $\beta(\mathrm{SE})$ & $\mathbf{p}$ & $\beta(\mathrm{SE})$ & $\mathbf{p}$ \\
\hline Intercept & $56.57(10.44)$ & & $11.20(4.54)$ & \\
\hline NF1 & $4.51(2.73)$ & 0.1013 & $1.21(1.23)$ & 0.3269 \\
\hline Attention Problems z-score (CBCL) & . & . & $-0.71(0.32)$ & 0.0263 \\
\hline Omissions percentile (CPT-II) & $-0.10(0.04)$ & 0.0235 & . & . \\
\hline Commissions percentile (CPT-II) & . & . & $0.05(0.02)$ & 0.0164 \\
\hline EVIP normalized score & . & . & . & . \\
\hline IQ & $0.26(0.09)$ & 0.0055 & $0.12(0.04)$ & 0.0047 \\
\hline Parents' education level & . & . & . & . \\
\hline Parents' socio-professional category & . & . & . & . \\
\hline Reading speed z-score (Alouette) & $6.77(1.83)$ & 0.0003 & $4.16(0.82)$ & $<.0001$ \\
\hline Reading accuracy z-score (Alouette) & $2.86(0.88)$ & 0.0015 & $1.43(0.40)$ & 0.0004 \\
\hline $\begin{array}{l}\text { Interaction: } \\
\text { Reading speed z-score (Alouette) x NF1 }\end{array}$ & $-5.13(2.21)$ & 0.0217 & $-2.75(1.00)$ & 0.0067 \\
\hline
\end{tabular}

$\beta$ : parameter estimates; SE: Standard Error; $\mathrm{p}$ : $\mathrm{p}$-value; $\mathrm{R}^{2}$ : coefficient of determination 
Among the attentional capacity measures, only the omissions score was associated with the text comprehension ability ( $\beta=-0.10 ; p=0.0235)$, while both CBCL "attention problem" and commission scores were associated with sentence comprehension $(\beta=-0.71 ; p=0.0263$ and $\beta=0.05 ; p=0.0164$ respectively). An increase in text omission difficulties in both the NF1 and control groups, lead to decreased text comprehension (with percentile omission increases of 10 points, text comprehension scores decreased by 1 point, when adjusted for other variables). Increases in attention difficulties assessed by CBCL, correlated with decreases in sentence comprehension (1SD in CBCL was associated with a 0.7 -point decrease in the sentence comprehension score when adjusted for other variables). With commission percentile increases of 10 points, sentence comprehension improved 0.5 points on average. Total IQ was strongly associated with both text and sentence comprehension $(\beta=0.26 ; p=0.0055$ and $\beta=0.12$; $\mathrm{p}=0.0047$, respectively) with a higher IQ correlating with better reading comprehension, adjusted for attention and reading measures. Regarding the "L'Alouette" reading test, good reading accuracy was associated, in all children, with good text and sentence comprehension $(\beta=2.86 ; p=0.0015$ and $\beta=1.43 ; p=0.0004$ respectively). Reading speeds affected text and sentence comprehension differentially in NF1 and non-NF1 groups (interaction tests $\mathrm{p}=0.0217$ and $\mathrm{p}=0.0067$ ), with faster reading associated with a larger improvement in reading comprehension in nonNF1 children, and only a small improvement in NF1 children (with speed increases of 1SD in Lobrot text and sentence scores, an increase of 6.77 and 4.16 points, respectively, in the non-NF1 population, and an increase of 1.64 and 1.14 points in the NF1 population, when adjusted for other variables). Parents' education level and socio-professional category were not significantly associated with reading comprehension and were removed from the two final models. Variables included in the final models accounted for $46 \%$ of variations in text comprehension scores and $57 \%$ of sentence comprehension variations, which is indicative of a good fit.

\section{Discussion}

The aim of our study was to investigate the association between attention problems and reading comprehension ability in children with NF1. We demonstrated the impact of attention problems on reading comprehension skills in children with NF1 and the importance of a multimodal evaluation of attention including direct and indirect tools.

\section{Neurofibromatosis Type 1 Challenges How Attention is Measured?}

We used an indirect parental assessment (checklist, CBCL) and a performance-based measure (CPT-II) of attention to determine whether attention processes affect reading skills. There was no significant concordance between these two assessment approaches, in the NF1 group and also not in the group of children without NF1. This could either be explained by a lack of relevance of at least one of these assessment tools or alternatively that the parental questionnaire and neuropsychological tests evaluate different dimensions.

\section{A Lack of Relevance of Parental Questionnaires or Objective} Measures of Attention: Results from previous research show that both indirect and direct measures of inattention, hyperactivity and impulsivity are appropriately validated, are viable $[47,48]$ and are complementary, both having their own advantages and disadvantages.

Most NF1 studies only use functional parental questionnaires (indirect measure) - possibly because they're easy and fast to administer, cost-effective, readily available, they cover the same numerous quantitative indices of child behaviors and are useful for assessing behaviors and responses for interventional purposes. The advantages of using such behavior-rating scales are well-described [51]: the standardization of asking questions (i) reduces the variability of the obtained information and ensures the relevance of the assessed behaviors, (ii) reduces subjectivity, judgments and therefore increases reliability. However, behavior-rating scales are also subject to significant bias [51,52,53] introduced by previous exposures which the raters may have had with a particular child (which vary among parents, teachers, friends, etc.), specific rater characteristics (e.g., depression) which may affect ratings provided, knowledge of the relevant and expected behavior, etc. The propensity for bias is also supported by a poor teacher and parent inter-rater agreement $[38,39]$. In addition, validations of behavior rating scales in epilepsy detected very low sensitivity (although specificity was high), especially for the Child Behavior Checklist [54,55]. The Rodenburg et al. (2005) review indeed identified that four out of the eleven items of the Attention Problems scale are ambiguous.

Direct behavioral performance-based data are more reliable than rating scales but are expensive, time-consuming, do not take into account the child's everyday environment [34], and can be difficult to administer to children as they rely on the child's willingness to cooperate.

Direct and Indirect Attention Assessment Tools: An Overlap? Although a few studies have performed both direct and indi- 
rect assessments and compared their consistency, these studies predominantly detected a weak correlation between the two approaches [49,56,57]. Direct and indirect tests measure different manifestations of inattention/attention and hyperactive/impulsive behaviors in different life settings: (1) indirect behavior attention teacher-rating scales measure behaviors observed in the classroom, (2) indirect behavior attention parent-rating scales (such as CBCL) measure the observed behaviors at home and during daily activities, whereas (3) direct measures of behaviors (such as CPT-II) are adapted to a child's capacity to attend to stimuli during a cognitive task. However, Ebert (2017) [58] found positive and significant convergence (partial correlation coefficients ranged from $r=.26$ to $r=.55$-medium to large-sized effects) between these types of tools, but without a complete overlap, indicating that both contribute diagnostic information but that they are not interchangeable. The current literature therefore confirm that parent (or teacher) reports and direct child assessments diverge and evaluate distinct functions. Indeed, the type of attention required for a child to focus on stimuli during a time-limited neuropsychological task significantly differs from that required to fulfill activities during daily life. It is also important to highlight that no study has to date attempted to determine which type of tool (parent report or direct child assessment) is more accurate to index specific domains of interest (i.e. the correlation between attention and reading).

\section{What about Direct and Indirect Attention Assessment in NF1 Specifically?}

Our findings are consistent with previous NF1 results that used neuropsychological tests in conjunction with functional questionnaire measurements [59]. Payne et al. (2011) [60] which found that questionnaires tap, more specifically, into real-world functions that are quite different from the cognitive assessment of the same function under standardized conditions (i.e. a quiet room) and highlight the importance of taking these two dimensions into account to guide remediation programs. Coutinho et al. (2016) [61] reported a low and often non-significant correlation between neuropsychological tests and parents' questionnaires, with the latter being strongly dependent on the positive or negative overall parental attitude. Gilboa et al. (2014) [34] advocates that direct behavior assessments cannot represent attention levels in NF1, because these measurements are too far removed from the reality of NF1 pathology and are therefore not representative of attention processes in real life. The authors therefore suggest that neuropsychological tests be adapted to the real world using virtual reality with "The Virtual Classroom" as a sensitive and ecologically valid assessment tool for use in the diagnosis of attention deficits in NF1.

Isenberg et al., (2013) [19] show that attention scores of NF1 children who satisfy the ADHD criteria are not significantly different from NF1 children without ADHD. The authors surmise that ADHD features defined in the rating scale do not fully capture all the attention performance deficits experienced by these children. They also state that parental identification of attention performance deficits underestimates the true prevalence of attention abnormalities.

In conclusion, no single test will capture all aspects of the complex attention domain in NF1 children, the preferable approach is therefore to combine performance-based and observer-rated outcome measures in a multimodal assessment of attention processes [62].

\section{Underlying Mechanisms of Attention on Reading Com- prehension in NF1}

\section{No Correlation, no Link, and Not Even the Same Processes:}

We detected a significant association between attention variables and reading comprehension when scored with direct and indirect tools, and in children with or without NF1. The contribution of attention components in reading-related activities has been previously demonstrated [63]. Children with ADHD may be more likely to show deficits in reading and other academic skills [64]. A study of school-aged children [65] has also revealed correlations between performance on the CPT-II, other behavioral ratings of inattention, and reading-related skills. In addition, Gray \& Climie (2016) [66] report successful medication treatments in children with both ADHD and RD. The association between attention and reading in non-NF1 children is well-accepted in a number of areas and implicates both decoding and comprehension [21,29]. With regards to comprehension, children with attention difficulties may present lower reading comprehension scores, difficulties in reporting the central idea of a text, and a proportional decrease in reading comprehension ability with the increasing length of the text read.

Although results from a number of studies indicate that attention and reading skills are interrelated and that both behavior-ratings and direct measures of inattention/attention are associated with reading skills, no study to date has directly examined whether these measures are unique (or overlapping) correlates of comprehension reading-related skills in children. In other words, how inattentive, hyperactive and impulsive behaviors relate to reading-related skills, measured via different methods (direct or indirect), remains an open question. In our study, we demonstrate that attention problems, assessed both objectively (CPT-II scores) and subjectively (parental questionnaires), do not cor- 
relate with the same reading comprehension domains (in both children with and without-NF1). Direct performance-based data (CPT-II, omission and commission scores) was significantly associated with text and sentence comprehension ability $(\mathrm{p}=0.0235$ and $\mathrm{p}=0.0164$ respectively), whereas indirect observer-rated of data (CBCL, checklist, attention problem scores) was only associated with sentence comprehension, both in NF1 and non-NF1 children. This confirms the complementarity of these evaluations and that children with and without NF1 would benefit from a multimodal assessment of attention skills. One of the reasons for this mixed picture is that reading comprehension tests can differ in their attentional demands [67]. In particular, the length of texts differs between tests (ranging from single sentences to long passages of text) or in the methods of assessment (response time taken into account or not). This can create differences in attentional load and thus influence the degree of correlation between attention tests. Secondly, the formats used to evaluate the association between attention and reading comprehension (i.e. texts and sentences) do not require the same attention resources. Consequently, texts and sentence reading comprehension are not correlated with the same attention tests and neither of the two types of tests (indirect and direct) alone can be recommended to assess reading comprehension. A multimodal assessment is a preferred approach.

\section{What about the Implication of Attention in Reading Compre- hension in NF1 Specifically?}

Attention is one of the primary cognitive concerns for NF1 children $[17,18,19]$. Although ADHD may affect the child's ability to learn [29], attention processes have received little consideration from the research community, to explain the reading deficit. Only one study [35] has investigated and showed a link between attention and academic achievement including reading, in NF1 (albeit by examining academic achievement rather than reading specifically). This reading score only assessed the reading of single words (Word Reading Spelling subtest of the WIAT) and therefore failed to score the complexity of the reading process. Furthermore, the precise nature of the association between reading and attention remains vague. In particular, the direction of causation cannot be inferred by the results obtained and is indistinguishable if Pride's results indicate that the presence of ADHD increases the presence of learning disabilities or vice versa. Indeed, the $\mathrm{ADHD}$ prevalence rate increased in NF1 children when other disorders such as Specific Learning Disabilities coexisted [4].

Overall, the literature to date is quite unclear on how ADHD affects cognitive functions in NF1. Furthermore, we believe that the precise nature of the association between attention and cognitive functions has not been adequately (and appropriately) studied. Indeed, all studies that have taken an interest in this question focused solely on evaluating NF1 alone or NF1 associated with ADHD (i.e. pathologic vs. non-pathologic ). As attention abilities are a continuum in NF1 (and all other populations), the best means to study attention problems and to determine their impact on academic achievement, is to analyze attention as a continuous variable rather than comparing groups with and without ADHD [59]. In accordance with the Lehtonen recommendation, our finding demonstrates the impact of attention level on reading comprehension level (and not only on ADHD). Our study therefore provides a strong argument supporting that attention difficulty in the NF1 child will have an impact on the child's academic performance and, particularly, on learning to read.

\section{Capturing Highly Specific Reading Skills: What Atten- tion Tests Need to be Used?}

To determine which were the greatest "predictors" of reading comprehension, we used models to explain text and sentence comprehension, obtained after elimination of variables procedure. This initially included both groups (NF1 and non-NF1 children), the CBCL "attention problem" $\mathrm{z}$-scores, both the omission and commission percentile scores of CPT-II, IQ, the reading speed and accuracy z-score of "L'Alouette", the EVIP normalized score, and the parents' socio-professional category and education level. Four notable results were obtained.

\section{No Single Measure Suffices as a Predictor of Reading Com-}

prehension: Firstly, and interestingly, no single attention measure can be considered a better or a unique reading comprehension predictor. Indeed, the final models retained, over the entire population of $139 \mathrm{NF} 1$ and control children - which explain $46 \%$ of the variance for text comprehension and $57 \%$ of the variance for sentence comprehension, indicate that the measure of inattention (CBCL and/or CPT-II omission), IQ (WISC-IV) and reading accuracy (CM "LAAlouette" index) have non-significant but different effects on the child's reading comprehension level (for both populations). This means that measures of inattention, IQ and reading accuracy are significantly associated together with the Lobrot efficiency in the understanding of text and sentences. When the measure of inattention increases, the measure of reading comprehension decreases, meaning that increases in attention problems are reflected in less efficient reading comprehension. When the measure of efficiency increases, the measure of reading comprehension increases, meaning that the higher the child's IQ, the better the child's understanding of the text. When the measure of reading accuracy increases, the measure of 
reading comprehension increases (Lobrot score), meaning that improvements in the child's reading accuracy, are reflected in an improvement in reading comprehension. To summarize, a good understanding of both sentences and texts is associated with a high IQ, good reading accuracy and few inattention problems. Reading comprehension is a complex process that requires the coordination of numerous cognitive abilities (in order to understand words, sentences, and texts), including phonological and orthographic knowledge, correspondence to letters, decoding, memory, etc., but also the general cognitive ability (IQ). Numerous studies have shown that reading is correlated with language abilities (verbal IQ), phonological awareness, working memory, and attention [64,68]. For IQ in particular, several studies have investigated the proportion of variance in reading ability that is explained by IQ [69]. However, even if an association is often evoked $[68,69,70]$, reading has an etiology that is not completely dependent on shared influences with IQ [69]. Furthermore, IQ is not often used in practice as a predictor of reading ability due to the existence of children with average reading ability and low IQ or average IQ and low reading ability (as in dyslexia). This implies that factors other than IQ are critical in the development of successful reading abilities, which is confirmed by our current study. Indeed, we report the exact nature, variance, and overlap between the contribution of components that affect reading comprehension. Our study therefore supports that reading is a multifaceted skill set and a complex process that encompasses reading accuracy, IQ and attention, and requires the implementation of several tools and evaluation approaches.

Difference between NF1 and non-NF1 Individuals: We showed that lower reading speeds are associated with a decrease in reading comprehension in both NF1 and non-NF1 populations, but that this association was stronger in the NF1 group. This may indicate that the effect of reading speed on reading comprehension is modulated by a third, as yet not identified factor, in the NF1 population.

\section{The Specific Role of Attention in Reading Comprehension:}

We found that attention level, rather than impulsivity or hyperactivity, is an effective predictor of the reading comprehension level in NF1 children as well as in non-NF1 children. This result is in line with previous studies $[20,66,71]$ that revealed that inattentive behaviors were strongly inversely associated with reading (particularly fluency and comprehension), but hyperactivity and impulsive behaviors did not predict reading achievement. However, these studies did not consider reading and attention as continuums and only considered the tail end of the normal curve (RD and ADHD as pathological conditions). They did not investigate the normal variation in the reading, attention interaction. In this sense, our findings confirm those obtained in earlier studies, showing the specific effects of attention on reading achievement when compared to impulsivity or hyperactivity. But they also improve on them, by highlighting for the first time, the definite role of attention in reading comprehension, even outside of its pathological forms.

We also surprisingly detected a correlation between reading comprehension and impulsivity. Indeed, we found that when the measure of impulsivity increased (CPT-II percentile commission), the measure of sentence reading comprehension decreased (Lobrot score). Here, the CPT-II commission had an effect on sentence reading comprehension (not significantly different between the 2 populations, NF1 and non-NF1). For example, an increase of 10 points in the commission score (impulsivity) led to an increase of 0.5 points in the Lobrot score (higher understanding). This result is not congruent with clinical reality. Firstly, children who were less impulsive presented a better analysis of the sentence or text and consequently a better comprehension. Secondly, it is most likely inattention rather than the hyperactivity that forms the association between ADHD and reading [22]. We therefore consider this result to be an artifact. Indeed, an elevated commissions score, as defined on the CPT-II, can be multifactorial: this score highlights, of course, a measure of impulsivity, but it is equally likely to indicate fast responses to the targets (in the CPT-II), resulting in an unstable impulsivity validity index in pediatric populations when it is associated with a test dependent on response time (as is the case for sentence comprehension). Consistent with this interpretation, the commissions score only correlated with the Lobrot sentence score results (which are timed, and where results depend on response times), and not with the Lobrot text score results (which are not timed, and where results do not depend on response times). The commission's score, in this case, appears to be a better marker of response time, than a measure of real impulsivity. We can also discuss the results of a recent study in which authors reported that impulsive behavior was positively related to reading skills [72]. More precisely in their study, individuals with a medium to a high level of hyperactivity and impulsivity but with a low level of inattention tended to perform better on reading measures than individuals with low-level hyperactivity and impulsivity but a medium level of inattention.

To summarize, (1) good sentence and text reading comprehension are not only associated with attentional measures but also with higher IQ, and good reading accuracy, irrespective of the population studied; (2) reading speed is also associated with good sentence and text comprehension in non-NF1 children, but not in NF1 children; (3) reading comprehension (text and 
sentence) depends on attention/inattention measures more than impulsivity/hyperactivity measures even if greater impulsiveness (measured by CPT-II commission) is associated with good sentence reading comprehension.

\section{Conclusion}

In the present report, we have focused on reading comprehension, attention assessment tools, in NF1 and non-NF1 children. When attention problems in NF1 children are analyzed as a continuum as opposed to two groups (ADHD and not ADHD), we found that inattention, rather than impulsivity and hyperactivity, correlates to the reading comprehension level in NF1 children. However, attention level is not the only parameter that has an impact on reading comprehension: IQ and the "L'Alouette" accuracy index indeed have an effect on reading comprehension, regardless of the population (NF1 and nonNF1).

There is an overall lack of consensus regarding how best to measure attention, and also whether both types of assessments (direct test-based and indirect questionnaire-based) may be used for the same purposes, especially in the context of investigating correlations with other cognitive measurements. We showed that both indirect and direct assessments are complementary to evaluate attention skills as they explore different domains. They are not interchangeable and should be used in parallel. The finding that these individual measurements demonstrate a unique association with a specific reading skill implies that both should be considered in the diagnosis process.

Moreover, the development of neuropsychological tests that are more sensitive to the child's test environment should be prioritized, particularly for evaluating NF1 children.

Acknowledgments: This work is dedicated to the children and families who agreed to participate in the study. We would like to thank the NF network France, ANR France, and the Occitanie Region for their support. The authors also thank Laurence Lion-François for her help and Petra Neufing for the English language editing.

Contributors' Statements: YC is the principal investigator of the study, conceived the idea for the study, and wrote the protocol and the manuscript. MB conceived the first working plan based on results and wrote the manuscript. ET carried out the statistical analysis, wrote the statistical sections of the manuscript, and reviewed the final manuscript. VLC contributed to the writing of the protocol and reviewed the final manuscript. NFM was involved in coordinating the study and carried out the neuropsy- chological tests. SL was involved in coordinating the study and in monitoring quality. SI and EP carried out the neuropsychological tests. PC, ES, ND, DR, IK, FR, MC, SB included and examined patients. EB reviewed the final manuscript and suggested a number of improvements. All authors approved the final manuscript as submitted and agree to be accountable for all aspects of the work.

Conflict of Interest: The authors have no conflicts of interest to disclose.

Funding Source: This work was supported by a grant from the Clinical Research Hospital Program from the French Ministry of Health (PHRC 2008, University Hospital of Toulouse, No. 0811301 ) and a grant from the Occitanie Region (APRTC No. 09004813).

Financial Disclosure: All the authors have no financial relationships relevant to this article to disclose.

Clinical Trial Registration: This study is registered with Clinical Trials.gov. number NCT02397967

\section{Contribution to the Field Statement}

Neurofibromatosis type 1 (NF1) is one of the most common childhood genetic disorders, affecting approximately 1 in 3000 individuals in the general population. A broad spectrum of cognitive deficits occurs in $30-70 \%$ of NF1 cases. Reading disorders are one of the primary concerns for NF1 children: $67 \%$ have problems in one or more reading subskills. Reading is a multifaceted skill and problems in this domain can result from difficulties in other areas, such as attention processes. Despite the majority of NF1 children experiencing attention difficulties, the impact of attention problems on reading comprehension has to date not been studied in the NF1 population. By considering attention and reading as continuums, a continuum ranging from good to poor readers and from good to poor attention skills in both NF1 and non-NF1 groups, we evaluated the effect of attention skills on reading comprehension, in NF1 and other populations with a developmental disorder (dyslexia). We also evaluated whether different types of tools (direct or indirect) can measure the same or different aspects of reading comprehension.

Children with or without NF1 ( $n=150 ; 8-12$ years of age), matched for age, gender, handedness, and reading level were submitted to direct and indirect attention tools as well as text and sentence reading comprehension. For both NF1 as well as non-NF1 children, attention capabilities greatly influenced 
reading comprehension, but there was only a weak correlation between direct and indirect attention measurements. Indirect observer-rated (questionnaires) and direct performance-based assessments of attention measure different components of reading skills. We make the case that children with NF1 would benefit from a multimodal assessment of attention skills, particularly where cognitive functions are evaluated to detect causal associations.

\section{References}

1) Evans D. G, Howard E, Giblin C, Clancy T, et al. (2010) Birth incidence and prevalence of tumor-prone syndromes: Estimates from a UK family genetic register service. American Journal of Medical Genetics, Part A, 152A: 327-332.

2) (1987) National Institutes of Health: Consensus Development Conference Statement: neurofibromatosis July 13-15, 6: 1-19. Bethesda, Md, USA.

3) Ferner RE (2007) Neurofibromatosis 1 and Neurofibromatosis 2: a twenty first century perspective. The Lancet Neurology 6: 340-351.

4) Hyman SL, Shores A \& North KN (2005) The nature and frequency of cognitive deficits in children with neurofibromatosis type 1. Neurology 65: 1037-1044.

5) North KN, Riccardi V, Samango-Sprouse C, Ferner R, et al. (1997) Cognitive function and academic performance in neurofibromatosis 1: consensus statement from the NF1 Cognitive Disorders Task Force. Neurology, 48: 1121- 1127.

6) Schrimsher GW, Billingsley RL, Slopis JM \& Moore BD (2003). Visual-spatial performance deficits in children with neurofibromatosis type-1. American Journal of Medical Genetics, 120A: 326-330.

7) Hyman SL, Shores A, North KN (2006) Learning disabilities in children with neurofibromatosis type 1: subtypes, cognitive profile, and attention-deficit-hyperactivity disorder. Developmental Medicine and Child Neurology 48: 973-977.

8) Chaix Y, Lauwers-Cancès V, Faure-Marie N, Gentil C, Lelong $S$, et al. (2018) Deficit in phonological processes: a characteristic of the neuropsychological profile of children with NF1. Child Neuropsychology 24: 558-574.

9) Graf A, Landolt MA, Capone Mori A, \& Boltshauser E (2006) Quality of life and psychological adjustment in children with neurofibromatosis type 1. The Journal of Pediatrics 149: 348-353. 10) Wolkenstein P, Rodriguez D, Ferkal S, Gravier H, Buret V, et al. (2009) Impact of neurofibromatosis 1 upon quality of life in childhood: a cross-sectional study of 79 cases. The British Journal of Dermatology 160: 844-848.

11) Coudé FX, Mignot C, Lyonnet S, \& Munnich A (2006) Academic Impairment is the Most Frequent Complication of Neurofibromatosis Type-1 (NF1) in Children. Behavior Genetics 36: 660-664.

12) Krab LC, Aarsen FK, De Goede-Bolder A, Catsman-Berrevoets CE, Arts WF, et al. (2008) Impact of neurofibromatosis type 1 on school performance. Journal of Child Neurology 23: 10021010

13) Cutting LE, Clements AM, Lightman AD, Yerby-Hammack, 
PD, \& Denckla MB (2004) Cognitive Profile of Neurofibromatosis Type 1: Rethinking Nonverbal Learning Disabilities. Learning Disabilities Research and Practice 19: 155-165.

14) Cutting LE, Koth CW, \& Denckla MB (2000) How children with neurofibromatosis type 1 differ from "typical" learning disabled clinic attenders: Nonverbal learning disabilities revisited. Developmental Neuropsychology17: 29-47.

15) Watt SE, Shores A, \& North KN (2008) An examination of lexical and sublexical reading skills in children with neurofibromatosis type 1. Child Neuropsychology 14: 401-18.

16) Orraca-Castillo M, Estévez-Pérez N, \& Reigosa-Crespo V (2014) Neurocognitive profiles of learning disabled children with neurofibromatosis type 1. Frontiers in Human Neuroscience 8.

17) Levine TM, Materek A, Abel J, O’Donnell M, \& Cutting LE (2006) Cognitive profile of neurofibromatosis type 1 . Seminars in Pediatric Neurology 13: 8-20.

18) Hachon C, Iannuzzi S, \& Chaix Y (2011) Behavioural and cognitive phenotypes in children with Neurofibromatosis type 1 (NF1): the link with the neurobiological level. Brain \& Development, 33: 52-61.

19) Isenberg J C, Templer A, Gao F, Titus JB, \& Gutmann DH (2013) Attention skills in children with neurofibromatosis type 1. Journal of Child Neurology 28: 45-49.

20) Sims DM \& Lonigan CJ (2013) Inattention, hyperactivity, and emergent literacy: different facets of inattention relate uniquely to preschoolers' reading-related skills. Journal of Clinical Child \& Adolescent Psychology 42: 208-219.

21) Willcutt EG, \& Pennington BF (2000) Comorbidity of reading disability and attention-deficit/hyperactivity disorder. Journal of Learning Disabilities 33: 179-191.

22) Zumberge A, Baker LA, \& Manis FR (2007) Focus on Words: A Twin Study of Reading and Inattention. Behavior Genetics 37: 284-293.

23) Samuelsson S, Lundberg I, \& Herkner B (2004) ADHD and Reading Disability in Male Adults. Journal of Learning Disabilities 37: 155-168.

24) Dally K (2006) The influence of phonological processing and inattentive behavior on reading acquisition. Journal of Educational Psychology 98: 420-437.

25) Walcott CM, Scheemaker A \& Bielski K (2009) A Longitudinal Investigation of Inattention and Preliteracy Development. Journal of Attention Disorders 14: 79-85.

26) Spira EG, \& Fischel JE (2005) The impact of preschool inattention, hyperactivity, and impulsivity on social and academic development: a review. Journal of Child Psychology and Psychiatry 46: 755-773.

27) Shaywitz B A, Fletcher JM, Holahan JM, Shneider AE, et al. (1995) Interrelationships between Reading Disability and Atten-
tion-Deficit/Hyperactivity Disorder, Child Neuropsychology1: 170-186,

28) Brock SE, Knapp PK (1996) Reading comprehension abilities of children with attention deficit/hyperactivity disorder. Journal of Attention Disorders 1: 173-185.

29) Miller AC, Keenan JM, Betjemann RS, Willcutt EG, Pennington BF, \& Olson RK (2013) Reading comprehension in children with ADHD: cognitive underpinnings of the centrality deficit. Journal of Abnormal Child Psychology 41: 473-483.

30) North K, Hyman S, \& Barton B (2002) Cognitive deficits in neurofibromatosis 1. Journal of Child Neurology, 17 605-612.

31) Koth CW, Cutting LE, \& Denckla MB (2000) The association of neurofibromatosis type 1 and Attention Deficit Hyperactivity Disorder. Child Neuropsychology 6: 185-194.

32) Roy A, Roulin JL, Charbonnier V, Allain P, Fasotti L, et al. (2010) Executive dysfunction in children with neurofibromatosis type 1: A study of action planning. Journal of International Neuropsychological Society, 16: 1056-1063.

33) Lidzba K, Granstrom S, Lindenau J, \& Mautner VF (2012) The adverse influence of attention deficit-disorder with or without hyperactivity on cognition in neurofibromatosis type 1 . Developmental Medicine and Child Neurology 54: 892-897.

34) Gilboa Y, Josman N, Fattal-Valevski A, Toledano-Alhadef H, \& Rosenblum, S (2014) Underlying mechanisms of writing difficulties among children with neurofibromatosis type 1 . Research in Developmental Disabilities 35: 1310-1316.

35) Pride NA, Payne J M, \& North KN (2012) The Impact of ADHD on the Cognitive and Academic Functioning o Children With NF1. Developmental Neuropsychology 37: 590-600.

36) Konold TR, Walthall JC, \& Pianta RC (2004) The behavior of child behavior ratings: Measurement structure of the child behavior checklist across time, informants, and child gender. Behavioral Disorders 29: 372-383.

37) Van der Oord S, Prins P, Oosterlaan J, \& Emmelkamp P (2006) The association between parenting stress, depressed mood and informant agreement in ADHD and ODD. Behaviour Research and Therapy 44: 1585-1595.

38) Angtrop I, Roeyer, H, Oosterlaan J, \& Van Oost P (2002) Agreement between parent and teacher ratings of disruptive behavior disorders in children with clinically diagnosed ADHD. Journal of Psychopathology and Behavioral Assessment 24: 6773.

39) Wolraich ML, Lambert EW, Bickman L, Simmons T, et al. (2004). Assessing the Impact of Parent and Teacher Agreement on Diagnosing Attention-Deficit Hyperactivity Disorder. Journal of Developmental \& Behavioral Pediatrics 25:41-47.

40) Rydell A (2009) Family factors and children's disruptive behaviour: an investigation of links between demographic charac- 
teristics, negative life events and symptoms of ODD and ADHD. Social Psychiatry and Psychiatric Epidemiology 45: 233-244.

41) Lefavrais P (2005) Reading analysis and dyslexia test (Alouette-R). Paris: ECPA.

42) Wechsler D (2005) Échelle d'intelligence de Wechsler pour enfants et adolescents - 4ème édition. Paris: ECPA.

43) Dunn LM Theriault-Whalen CM \& Dunn LM (1993) Échelle de vocabulaire en images Peabody. Adaptation française du Peabody Picture Vocabulary Test - Revised Pearson: Canada.

44) Jacquier-Roux M, Valdois S, Zorman M, Lequette C, \& Pouget G (2005) Odedys, a screening tool for dyslexia. Version 2.

45) Lobrot M (1973) Read tests to assess reading ability and spelling level. Paris: E.S.F.

46) Lindgren SD \& Benton AL. (1980) Developmental Patterns of Visuospatial Judgment. Journal of Pediatric Psychology 5: 217-225.

47) Conners CK \& MHS Staff (Eds.) (2000) Conners' Continuous Performance Test II: Computer Program for Windows Technical Guide and Software Manual. North Tonwanda, NY: Mutli-Health Systems.

48) Achenbach TM \& Rescorla, LA (2001) Manual for the ASEBA school-age forms and profiles. Burlington, VT: University of Vermont, Research Center for Children, Youth, and Families.

49) Epstein JN, Erkanli A, Conners CK, Klaric J, et al. (2003)

Relations between continuous performance test measures and ADHD behaviors. Journal of Abnormal Child Psychology 31: 543-554.

50) Youngwirth SD, Harvey EA, Gates EC, Hashim RL. \& Friedman-Weieneth JL (2007) Neuropsychological Abilities of Preschool-Aged Children Who Display Hyperactivity and/or Oppositional-Defiant Behavior Problems. Child Neuropsychology 13: 422-443.

51) Edelbrock C (1990) The Child Attention Problems Scale. In: Barkley RA, editor. Attention Deficit Hyperactivity Disorder: A Handbook for Diagnosis and Treatment. Guilford 302-305.

52) Mandal RL, Olmi DJ \& Wilczynski S M (1999) Behavior rating scales: Concordance between multiple informants in the diagnosis of attention-deficit/hyperactivity disorder. Journal of Attention Disorders, 3: 97-103.

53) Yoder P \& Symons F (2010) Observational measurement of behavior. New York, NY, US: Springer Publishing Co.

54) Perrin E C, Stein REK, \& Drotar D (1991) Cautions in Using the Child Behavior Checklist: Observations Based on Research About Children with a Chronic Illness. Journal of Pediatric Psychology, 16: 411-421.

55) Rodenburg R, Stams GJ, Meijer AM, Aldenkamp AP \& Deković M (2005) Psychopathology in Children with Epilepsy: A
Meta-Analysis. Journal of Pediatric Psychology 30: 453-468.

56) Egeland J, Johansen SN \& Ueland T (2009) Differentiating between ADHD sub-types on CPT measures of sustained attention and vigilance. Scandinavian Journal of Psychology 50: 347-354.

57) McGee R, Clark SE, \& Symons DK (2000) Does the Conners' Continuous Performance Test Aid in ADHD Diagnosis? Journal of Abnormal Child Psychology 28: 415-424.

58) Ebert KD (2017) Convergence between parent report and direct assessment of language and attention in culturally and linguistically diverse children. PLoS ONE 12: e0180598.

59) Lehtonen A, Howie E, Trump D, \& Huson SM (2013) Behaviour in children with neurofibromatosis type 1: cognition, executive function, attention, emotion, and social competence. Developmental Medicine and Child Neurology 55: 111-125.

60) Payne JM, Hyman SL, Shores EA, \& North KN (2011) Assessment of executive function and attention in children with neurofibromatosis type 1: Relationships between cognitive measures and real-world behaviour. Child Neuropsychology 17: 313-329. 61) Coutinho V, Kemlin I, Dorison N, Billette de Villemeur T, Rodriguez D, \& Dellatolas G (2016) Neuropsychological evaluation and parental assessment of behavioral and motor difficulties in children with neurofibromatosis type 1. Research in Developmental Disabilities 48: 220-230.

62) Walsh KS, Janusz J, Wolters PL, Martin S, Klein-Tasman BP, REiNS International Collaboration (2016). Neurocognitive outcomes in neurofibromatosis clinical trials: Recommendations for the domain of attention. Neurology 87: S21-S30.

63) Pham AV, Fine JG, \& Semrud-Clikeman S (2011) The Influence of Inattention and Rapid Automatized Naming on Reading Performance. Archives of Clinical Neuropsychology 26: 214224.

64) Commodari E, \& Guarnera M (2005) Attention and Reading Skills. Perceptual and Motor Skills 100: 375-386.

65) Lam CM, \& Beale IL (1991) Relations Among Sustained Attention, Reading Performance, and Teachers' Ratings of Behavior Problems. Remedial and Special Education 12: 40-47.

66) Gray C, \& Climie EA (2016) Children with Attention Deficit/ Hyperactivity Disorder and Reading Disability: A Review of the Efficacy of Medication Treatments. Frontiers in Psychology, 07.

67) Keenan JM, \& Meenan CE (2014) Test differences in diagnosing reading comprehension deficits. Journal of Learning Disabilities 47: 125-135.

68) Gayan J, \& Olson RK (2001) Genetic and environmental influences on orthographic and phonological skills in children with reading disabilities. Developmental Neuropsychology 20: 487-511. 
69) Harlaar N, Hayiou-Thomas ME, \& Plomin R (2005) Reading and general cognitive ability: a multivariate analysis of 7-yearold twins. Scientific Studies of Reading 9: 197-218.

70) Tiu RD, Thompson LA \& Lewis BA (2004) The Role of IQ in a Component Model of Reading. Journal of Learning Disabilities 36: 424-436.

71) Pham AV (2013) Differentiating Behavioral Ratings of Inattention, Impulsivity, and Hyperactivity in Children: Effects on Reading Achievement. Journal of Attention Disorders 20: 674683.

72) Tymms P \& Merrell C (2011) ADHD and academic attainment: Is there an advantage in impulsivity? Learning and Individual Differences 21: 753-758.

Submit your manuscript to a JScholar journal and benefit from:

ब Convenient online submission

ฯ Rigorous peer review

q Immediate publication on acceptance

ฯ Open access: articles freely available online

I High visibility within the field

I Better discount for your subsequent articles

Submit your manuscript at http://www.jscholaronline.org/submit-manuscript.php 